\title{
Abordar lo complejo desde el diseño: una mirada hacia la transdisciplinariedad*
}

\author{
Leonardo Andrés Moreno Toledano ${ }^{1}$ \\ (D) $h t t p: / / o r c i d . o r g / 0000-0002-9447-6362$
}

Universidad Autónoma de Ciudad Juárez, Chihuahua, México

DOI: http://dx.doi.org/10.17081/eduhum.19.33.2650

Recibido: 18 de junio de 2016

Aceptado: 10 de abril de 2017

\section{Approaching complexity from the design: a look at the transdisciplinary}

Palabras clave:

Complejidad,

Transdisciplinariedad, Diseño,

Investigación, Sostenibilidad.

Key words:

Complexity, Transdisciplinarity,

Design, Research, Sustainability.

\begin{abstract}
Resumen
El presente ensayo plantea una reflexión sobre la dificultad que las disciplinas actuales presentan al enfrentarse a los problemas complejos de nuestra era. Para ello, se expone la necesidad de abordar los fenómenos actuales desde enfoques no tradicionales y desarrollados a raíz de algunas teorías que han sido creadas con el fin de explicar este tipo de problemas. Asimismo, en el artículo se resalta el potencial del diseño, como disciplina orientada a la práctica, para encontrar soluciones a diversos fenómenos que se suceden en nuestras sociedades a partir de visiones no unidisciplinares.
\end{abstract}

\begin{abstract}
The present essay raises a reflection on the difficulty that nowadays disciplines present when facing the complex problems of our era. From this, it exposes the need to address current phenomena from non-traditional approaches through the exploration of some theories that have been developed in order to explain this type of problems. It also proposes the potential of design, as a discipline oriented to practice, to help in the search for solutions to various phenomena that occur in our societies from non-unidisciplinary visions.
\end{abstract}

Referencia de este artículo (APA): Moreno, L. (2017). Abordar lo complejo desde el diseño: una mirada hacia la transdisciplinariedad. En Revista Educación y Humanismo, 19(33), 369-385. http://dx.doi.org/ 10.17081/eduhum.19.33.2650

\footnotetext{
* Artículo vinculado a la línea de investigación Diseño para la sostenibilidad del Cuerpo Académico CA116 Diseño, Usuario y Entorno, adscrito a la Universidad Autónoma de Ciudad Juárez, Chihuahua, México.

1. Profesor-Investigador adscrito al departamento de diseño de la Universidad Autónoma de Ciudad Juárez, Doctor en Creación y Teorías de la Cultura (UDLAP), Maestro en Diseño Holístico (UACJ), Líder del CA116 Diseño, Usuario y Entorno (UACJ), Miembro del Sistema Nacional de Investigadores (Conacyt, México). lemoreno@uacj.mx
} 


\section{Introducción}

Actualmente no se requiere mucho esfuerzo para entender que el mundo que habitamos es complejo. De hecho, en él predomina el cambio y se observa cada vez más la incertidumbre, la pluralidad social y cultural y el caos, lo que en conjunto influye en nuestra vida cotidiana. Empero, el reconocimiento de nuestro entorno como algo complejo y el estudio de las relaciones que se desarrollan entre los diversos elementos que lo conforman, así como la manera en que estos construyen y transforman nuestro conocimiento y el modo en que nos desenvolvemos tanto individual como colectivamente, es algo relativamente nuevo en el ámbito de las disciplinas científicas. Apenas en los últimos años, hemos comenzado a entender que necesitamos hacer cambios en la manera en que abordamos y estudiamos nuestro entorno, especialmente cuando se trata de la búsqueda de soluciones a problemas complejos como las crisis económicas, la pobreza, la migración o la creciente inseguridad y violencia en nuestras calles. Lo anterior ha llevado a algunos estudiosos a afirmar que nuestro "mundo se ha mostrado mucho más complejo de lo que pretendía ser, y dicha complejidad exige que aquellas formas con que se creía que era posible conocerlo, sean revisadas profundamente y adecuadas a las actuales demandas de la sociedad global" (Flores \& Millán 2002, p.6).

En un sentido similar, Brown, Harris y Russel (2010) plantean que la imaginación [relacionada con la creatividad, la visión, la innovación y la originalidad] es el primer requerimiento para lidiar con lo paradójico, la incertidumbre y lo complejo. "En un sentido práctico, esta debe ser algo central para cualquiera que se involucre en cambiar la sociedad en que vive" (p.6). Lo anterior deja a las disciplinas creativas como las del diseño en una importante posición, dada su vocación hacia la innovación. De este modo, lo que plantean Brown, Harris y Russel, al igual que Edgar Morin y Basarab Nicolescu, es la necesidad de buscar respuestas a las problemáticas de nuestro mundo contemporáneo de manera creativa, mediante acercamientos distintos a los de la tradición positivista y, para ello, un elemento imprescindible será el diálogo entre disciplinas (Feo, 2015, p.221). Cabe mencionar que lo anterior no niega los aportes que el trabajo disciplinar ha brindado y continuará haciendo en nuestra sociedad, pero sí plantea que dicho enfoque tiene una visión limitada de los objetos que estudia (Moreno, 2013, p.23). Podemos decir, entonces, que aun cuando las diversas disciplinas -incluidas las áreas de diseño- sean capaces de responder a ciertas problemáticas de carácter específico, cuando nos encontramos ante un problema complejo como los anteriormente mencionados, el conjunto de variables [si observamos el problema en todo su contexto] y las relaciones entre estas, pueden ser tan amplias que es imposible abordarlas y darles respuesta desde una perspectiva unidisciplinar. En efecto, cuando nos encontramos ante un objeto complejo, nos hallamos con la dificultad de describirlo o explicarlo, ya que por la misma razón admite numerosas dimensiones, trazos diversos o indistinción interna. Por ello, se requiere de una nueva 
lente para abordar las complejidades de nuestro mundo actual, que también debe orientarse hacia la resolución de problemas. Esta nueva mirada es definida con nombres como enfoques multidisciplinares, interdisciplinares o transdisciplinares, los cuales, es importante mencionar, no deben ser entendidos como sinónimos, sino como niveles distintos de integración entre disciplinas.

\section{La complejidad en las Ciencias Sociales y} las Humanidades

$\mathrm{Si}$ bien, los principales argumentos del pensamiento complejo pueden ser comprendidos a partir de la visión científica, sociológica y filosófica de Edgar Morin (1994), aclarar su paso por las diversas áreas del conocimiento, desde su surgimiento en las ciencias clásicas a su aplicación en las Ciencias Sociales y las Humanidades, requiere de una aproximación distinta. En este sentido, un acercamiento sobre las diversas ciencias de la complejidad puede sernos útil para obtener un panorama más amplio sobre su influencia actual en los diversos saberes de la humanidad. Pero en todo caso, conviene decir que en este trabajo no se profundiza en cada una de ellas, sino que solamente se exponen de manera breve, con el fin de mostrar su relación con las ciencias humanas.

Como se ha afirmado, el interés actual en la complejidad es reciente. Los primeros institutos orientados a su estudio surgieron a finales de los años setenta del siglo pasado, así, en 1978, se creó el Centro de Estudios para la Dinámica
No-Lineal en el Instituto La Jolla (CA, USA); a comienzos de los años ochenta, el Instituto Santa Cruz para la ciencia No-Lineal (CA, USA), así como el Centro para estudios No-Lineales en el Laboratorio Nacional de los Álamos en Nuevo México (USA); y en 1984, surge el Instituto Santa Fe (NM, USA), considerado como el más famoso de los centros para estudios No-Lineales (Maldonado \& Gómez, 2010, p.9). Por tanto, se puede decir que aunque hace poco tiempo se ha establecido el paradigma de la complejidad y que falta mucho para que pueda considerarse como el arquetipo vigente, sí se observa que el interés que genera es un territorio fructífero, lo cual se aprecia en el creciente número de publicaciones y eventos realizados en los últimos treinta años (p.9).

En sus inicios, tanto las Ciencias Sociales como las Humanidades mostraban un lenguaje altamente permeado por las ciencias consideradas más sólidas: matemáticas, física, química y biología. Pero, desde mediados del siglo XIX se encontraron y describieron fenómenos que escapaban de los paradigmas hasta entonces establecidos por dichas ciencias y cuyos comportamientos divergían de las leyes de la predictibilidad. Un ejemplo de lo anterior fue la realidad subatómica cuando se descubrió que el átomo no es un elemento unitario, irreductible e indivisible, sino un sistema formado por diversas partículas y sus interacciones. A partir de este tipo de fenómenos, se comienza a observar la insuficiencia de las ciencias clásicas en la comprensión de ciertos sistemas: tanto 
naturales [clima, universo, etc.] como creados por el hombre [economía, transporte, ciudad, etc.], y se impone, a partir de ello, la necesidad de considerar no solo las partes sino la relación entre estas y su entorno, lo que requiere, como ya se mencionó, de nuevas aproximaciones. Lesbia Payares (2011) evidencia dichos cambios al explicar que "[...] un universo cuántico comienza a emerger, produciendo un profundo cambio en nuestra visión del mundo, y determinando el paso de la concepción reduccionista, mecanicista, cartesiana, newtoniana y lineal de la simplicidad, a una visión no lineal, hologramática e integradora de la complejidad" (p.23).

Para abordar dichos fenómenos, algunos científicos comenzaron a desarrollar -desde las mismas ciencias exactas- diversas teorías que fueron evolucionando hasta convertirse en lo que actualmente conocemos como ciencias de la complejidad. La primera de ellas fue la denominada Teoría del Caos, desarrollada por Edward N. Lorenz entre los años 1962 y 1964. Lorenz, en efecto, fue el primero en reconocer el comportamiento caótico de un sistema; descubrió que pequeñas diferencias en un medio dinámico como la atmósfera terrestre podían desencadenar en muchas ocasiones resultados inesperados. Estas observaciones lo llevaron a formular lo que hoy se conoce como el efecto mariposa, un término usado para explicar que pequeños cambios sucedidos en un sistema dinámico pueden producir comportamientos insospechados. Sus hallazgos marcaron el comienzo de nuevas áreas de estudio, no solo en las matemáticas, sino también en las ciencias biológicas, sociales y físicas. $\mathrm{Al}$ respecto, Fernando Almarza (2002) explica que:

Las dinámicas sistémicas del caos han generado la necesidad de nuevos conceptos y técnicas de experimentación, con gran incidencia en la elaboración de sistemas de representación de la realidad y sus bases filosóficas, metafísicas y metodológicas acerca del significado de la impredecibilidad e inestabilidad compleja en los procesos naturales, culturales y sociales, así como de sus comportamientos posibles a largo plazo. Su extrapolación a otros dominios del conocimiento humano es aplicada en economía, sociología, teoría cultural, neurociencia, planificación urbana y música, entre otras. (p.2)

Un ejemplo de la extrapolación a otros dominios del conocimiento humano por parte de la teoría del caos, se encuentra en el ensayo Welcome to Cyberia: Notes on the Anthropology of Cyberculture, escrito por Arturo Escobar et al. (1994). En él, se discute acerca de nuevos conceptos y metáforas que estaban abriéndose paso en la ciencia y la tecnología moderna:

[...] Los científicos que trabajan en el desarrollo de la ciencia de la complejidad no dudan de que están en el umbral de una gran revolución científica. En vez de enfatizar la estabilidad en la naturaleza y en las sociedades, enfatizan las inestabilidades y las fluctuaciones; en lugar de procesos lineales 
reversibles, la no linealidad y la irreversibilidad se sitúan en el centro de la investigación científica. De manera similar, los "sistemas conservadores" (sistemas físicos considerados aislados de su entorno) han dado paso a sistemas "autoorganizados", equilibrio estático, equilibrio dinámico y no equilibrio, orden al caos, elementos fijos y cantidades a patrones y posibilidades, y predicción a la explicación. (p.221)

Asimismo, Escobar explica que "el caos debe entonces ser visto como una fuerza que se negocia en diversos sitios dentro de la cultura, incluyendo la ciencia, el postestructuralismo y el posmodernismo; es parte de la condición posmoderna, ya sea reflejada en la literatura, las ciencias humanas o las ciencias de la complejidad" (p.222). Como se puede apreciar, la argumentación de Escobar se encuentra directamente ligada con el rompimiento de los meta-relatos. Es decir, con la idea relativa al final de las grandes narrativas de la modernidad (Lyotard, 1987).

Otra teoría de gran importancia en el desarrollo de las ciencias de la complejidad es la Geometría de Fractales desarrollada por Benoit Mandelbrot (1967). Para este autor, un fractal es un objeto geométrico en el que se repite el mismo patrón a diferentes escalas y con diferente orientación. Algunos ejemplos de ello son: la hoja de un helecho, el brócoli o la organización de las semillas de girasol. La teoría de fractales tuvo su origen en la necesidad de conocer de manera exacta la longitud de la costa de Ingla- terra. Mandelbrot se interesó en ello después de conocer las investigaciones desarrolladas por el físico y meteorólogo inglés Lewis F. Richardson (1881-1953), quien se había sorprendido al encontrar una discrepancia de hasta un $20 \%$ en las medidas de las fronteras entre España, Portugal, Bélgica y Holanda, lo que suscitaba el interrogante de que si todos medían la misma costa con el mismo sistema métrico, ¿por qué existían dichas discrepancias? Richardson concluyó que no existe una manera exacta de medir una costa, lo que se conoce actualmente como The Coastline Paradox.

A partir de lo anterior, Mandelbrot escribió un artículo titulado precisamente "¿Qué longitud tiene la costa de Inglaterra?" (1967), en el que se preguntaba si la costa de Inglaterra mediría lo mismo si la miramos desde un satélite en el espacio o desde un aeroplano, o paseando por la playa en bicicleta o midiendo el perímetro de cada grano que la componía. De esta manera, Mandelbrot desarrolló su teoría para explicar la manera en que se podrían medir este tipo de objetos. Desde entonces, la teoría de fractales se ha aplicado en diversas disciplinas de las Ciencias Sociales, como la psicología y la sociología.

En el recorrido por las ciencias de la complejidad, también encontramos la Teoría de Catástrofes, desarrollada por René Thom entre 1968 y 1972 para designar los cambios súbitos, imprevistos e irreversibles que se sucedían en ciertos fenómenos. Sobre ello, Miguel Espinoza explica que: 
La forma no puede tener otra explicación que geométrica y dinámica. El mundo consiste, en última instancia, en formas y fuerzas, y toda tentativa de eliminar unas $\mathrm{u}$ otras no puede sino fracasar. Por eso, en la metodología de las catástrofes, Thom combina los medios de los sistemas dinámicos [génesis dinámica de las formas] y de la topología diferencial [génesis estática de las formas] para explicar las “catástrofes" o discontinuidades de las regiones donde se producen cambios bruscos de estado, fronteras o bordes de los sólidos, transiciones de fase, etc. La catástrofe es el lugar del sistema donde este pasa abruptamente de un estado a otro, aunque -y esta observación es importante- los factores externos que controlan el proceso cambian de manera continua. (p.323)

Miguel Outerelo (2000) agrega que a partir de 1974 la teoría de catástrofes comenzó a popularizarse, dando así inicio a la publicación de centenares de artículos científicos con las más variadas aplicaciones:

El estudio de los latidos del corazón, el estudio de los impulsos nerviosos, el estudio de la anorexia nerviosa, la gastrulación y formación de somitas en anfibios y aves, la embriología, el comportamiento inestable de la bolsa, los conflictos causados por el estrés, los motines de las cárceles, la estabilidad de los barcos, la óptica física y geométrica, la lingüística, la teoría elemental de partículas y un largo etcétera. (p.254)
A comienzos del nuevo milenio se dio paso a otra importante ciencia de la complejidad: la Teoría de Redes Complejas, que fue desarrollada por D. Watts, L. Barabási y S. Strogatz entre los años 2001 y 2003. En este sentido, aunque la teoría de grafos, que explica la relación entre la cantidad de puntos de conexión existente entre dos o más nodos, se sitúa a mediados de los años cincuenta del siglo pasado, a partir de las publicaciones de Erdös y Rényi; no fue sino hasta finales de siglo, con la introducción del modelo de red de mundo pequeño de Watts y Strogatz, así como el de Barabási y Albert, que se desarrolla un interés exponencial en las redes complejas. El objetivo de Barabási (2002) consistía en explicar que todo se encontraba conectado con todo lo demás y la relación que tenía esto con la ciencia, los negocios y la vida cotidiana. Para él, las redes podían ser encontradas en cualquier cosa; desde las células cancerígenas hasta las economías globales, de ahí que todos los objetos que componen una red deban ser vistos en relación a nodos y a las conexiones surgidas entre estos. Un concepto muy útil en la teoría de Barabási es el de conector, que es básicamente entendido como un gran número de conexiones (p.55). Este concepto puede ser extrapolado fácilmente a las redes sociales y a la ciudad vista como red, es decir, como un conjunto de nodos de actividad -lugares de trabajo, hogares, estaciones de transporte, instituciones y espacios públicos- en los que se desarrollan múltiples y diversas conexiones que generan un alto grado de complejidad. 
Las teorías hasta aquí mencionadas propiciaron de manera acelerada el interés por la complejidad en áreas de las ciencias que anteriormente no eran estudiadas desde dicha perspectiva.

En el caso de las Ciencias Sociales, según explica Alberto Flores (2002), surgen:

[...] a partir de mediados del siglo $\mathrm{XX}$ toda una serie de circunstancias como el cambio en el ordenamiento geopolítico del mundo, la descolonización, la aparición de nuevos actores sociales y la creciente difusión de las tecnologías de la información, [que] entre otros, comenzaron a afectar la disciplinarización de las ciencias sociales y a cuestionar el tipo de conocimiento, hasta entonces hegemónico, que dichas estructuras producían. Es dentro de dicho contexto que empiezan a surgir nuevos campos para abordar los fenómenos sociales, como los estudios culturales o los estudios poscoloniales, entre otros, que pretenden abarcar cuestiones tales como los problemas de género, estudios "no-eurocéntricos", la importancia de lo local y lo histórico, y/o el reconocimiento de valores asociados al desarrollo tecnológico. Estos nuevos campos no se constituyen como nuevas disciplinas, sino como una especie de espacio más allá de las disciplinas que ha contribuido a desestabilizar la división tradicional del conocimiento. (p.7).

Por lo anterior, Maldonado y Gómez (2010) afirman que en la actualidad el "fenómeno de máxima complejidad conocida en el universo lo constituyen, sin lugar a dudas, los sistemas vivos, la vida" (p.57). Y agregan que esta idea ya se veía reflejada en la teoría de Bertalanffy, quien planteaba que las Ciencias Sociales y Humanas "[...] poseen, estudian, se ocupan de los sistemas, fenómenos y comportamientos de máxima complejidad conocida hasta la fecha" (p.59).

Así, podemos puntualizar, que lo complejo en lo social surge y se hace más evidente a partir del postmodernismo, esto es, a mediados de los años sesenta. En este marco el postestructuralismo nace como un pensamiento filosófico que pretende trascender los límites del estructuralismo y, como tal, niega la individualidad y el acontecimiento, introduciendo términos como la discontinuidad, la diferencia, lo aleatorio, lo fortuito y la diseminación. Con ello se genera "una ruptura epistemológica que se convierte en una brecha insalvable con el acontecimiento de mayo del 68 en Francia" (García, 2009, s/p). Sus principales protagonistas fueron Jacques Derrida, Michel Foucault y Roland Barthes, a quienes más tarde seguirían algunos filósofos franceses como Jean-François Lyotard y Gillez Deleuze. Este último, en conjunto con el psicoanalista Félix Guattari plantean un concepto que resulta de gran importancia para acercarnos en la comprensión sobre la manera en que la complejidad se presenta en las sociedades contemporáneas: el rizoma. Deleuze y Guattari construyen su pensamiento alrededor del modelo de rizoma 
[multiplicidad], en contraproposición con el modelo de pensamiento arbóreo. El modelo arbóreo plantea una imagen del mundo surgida de una lógica lineal [tronco], que representa la física, y una ramificación dicotómica [otros saberes] que brota de una misma raíz [metafísica] (Velázquez, 2010, p.55). El rizoma, en cambio, se encuentra hecho de muchas dimensiones, de direcciones cambiantes: "de multiplicidades, de líneas, estratos y segmentaridades, líneas de fuga e intensidades" (Deleuze \& Guattari, 2002, p.10).

Para Deleuze y Guattari, el rizoma, a diferencia de los árboles o de sus raíces:

[...] conecta cualquier punto con otro cualquiera, cada uno de sus rasgos no remite necesariamente a rasgos de la misma naturaleza; el rizoma pone en juego regímenes de signos muy distintos e incluso estados de no-signos. [...] No se deja reducir ni a lo uno ni a lo múltiple $[\ldots]$ contrariamente a una estructura, que se define por un conjunto de puntos y posiciones [...] el rizoma solo está hecho de líneas $[\ldots]$ se relaciona con un mapa que debe ser producido, construido, siempre desmontable, conectable, alterable, modificable, con múltiples entradas y salidas, con sus líneas de fuga. (p.25)

Asimismo, para Deleuze y Guattari el rizoma se encuentra conformado de mesetas, que, siguiendo a Barabási, son entendidas como regiones en las que se generan continuas inten- sidades que se conectan con otras a través de líneas y nodos.

De acuerdo con lo visto hasta aquí, se puede afirmar que la complejidad como paradigma ha sido adoptada, en mayor o menor medida, por la diversidad de áreas de conocimiento que integran el conocimiento humano, desde la física, donde nace, la biología y las matemáticas, hasta las Ciencias Sociales, los negocios, la religión, la psicología, la antropología, la historia, etc. Así pues, hoy en día es dable sostener que la complejidad es parte de nuestra cultura, y de alguna manera siempre lo ha sido.

\section{Disciplinas e indisciplinas en el saber}

Ahora bien, el abordaje de la complejidad requiere observar los problemas desde miradas distintas a las planteadas por el modelo cartesiano, por lo que, a continuación, describiremos brevemente las características de los principales enfoques:

Lo que conocemos como enfoque disciplinar o unidisciplinar es una aproximación a la práctica y la investigación, la cual consiste en que una sola disciplina sea la encargada de estudiar o resolver los problemas de una sociedad, siendo esta la manera normal o tradicional de resolución de problemas. Su objetivo principal es producir conocimiento teórico sobre la física y la naturaleza humana. El reflejo de su organización se encuentra normalmente en las universidades, a partir de sus áreas y departamentos. Por otra parte, sus sistemas de control 
de calidad se desarrollan a partir de la revisión de pares y los resultados de sus investigaciones se publican en revistas especializadas. Así, un campo disciplinar puede ser definido como un grupo de personas que siguen los mismos objetos de estudio, tratando de responder a un conjunto específico de preguntas de investigación y compartiendo los mismos paradigmas, metodologías, conceptos, técnicas y teorías (Kuhn, 1962; Boradkar, 2010). Contrario a los enfoques disciplinares, los enfoques no unidisciplinares envuelven la participación de más de un área de conocimiento y su objetivo mayor es la aplicación de estos en la resolución de problemas complejos. Estos enfoques se caracterizan por orientarse hacia la práctica, por lo que su objetivo no es tanto descubrir las leyes o principios de la naturaleza, sino el estudio de los sistemas complejos.

Según Robinson (2008), quienes practican este tipo de enfoques:

$[\mathrm{N}]$ o se encuentran al margen entre disciplinas sino en ocasiones se encuentran al margen de la academia, están familiarizados con que las problemáticas del mundo real que buscan solucionar no se pueden expresar fácilmente en términos del conocimiento disciplinar, frecuentemente son críticos de las disciplinas, típicamente se encuentran más interesados en crear formas de conocimiento útiles que en la creación de nuevas disciplinas, trabajan con los compañeros y las audiencias como coproductores y su propósito es más el de navegar entre las disciplinas para lograr un propósito particular que la de llenar los huecos entre ellas. (p.72)

Si bien históricamente hablando, la idea de síntesis, del pensamiento holístico y del conocimiento unificado estaban presentes desde la Grecia antigua, el uso de términos no unidisciplinares durante la era moderna puede encontrarse en distintos momentos. Un ejemplo de ello es la visión de la unificación de la ciencia presentada por Augusto Comte entre los años 1830 a 1842, y publicada en su Curso de filosofía positivista. En él, Comte planteaba la necesidad de proporcionar a las mentalidades individuales un sistema de creencias para unificar el espíritu colectivo. Es decir, a partir del estudio de las cosas individuales, pretendía conocer el todo, unificarlo. Algo similar pero más concreto, fue lo ocurrido con la fundación del Social Science Research Council, creado en 1923 con la intención de realizar ciertas investigaciones que cruzaran dos o más de los campos disciplinares que constituían el consejo -antropología, economía, ciencias políticas, psicología y sociología- a partir del intercambio de ideas, métodos y técnicas entre estos. La temprana formación de disciplinas hibridas en la década de los treinta, como la psicología social, la sociología política y la antropología social, son ejemplos de ello (Thompson, 2010, p.22). Asimismo, algunos reconocen este tipo de aproximaciones en las décadas de los sesenta y los setenta, a partir de un cambio en la educación y la experimentación sucedido durante las pláticas de Jean 
Piaget, Erich Jantsch y André Lichnerowicz en el taller internacional InterdisciplinariedadProblemas de la Enseñanza e Investigación en las Universidades (1970), financiado por la Organización Económica para la Cooperación y el Desarrollo (OCDE) (Nicolescu, 2006 p.15). El interés surgido desde entonces ha llevado a la convicción de que hoy los enfoques no unidisciplinares son básicos, tanto para la educación como para la innovación (Thompson, 2010, p.1). Estos permiten al individuo relacionarse con otras áreas del conocimiento y ampliar su información sobre algún concepto o problema, con lo que obtienen un panorama distinto de las cosas $\mathrm{y}$, por ende, una visión más amplia, creativa e innovadora que le habilita para buscar soluciones más allá de su propia especialidad.

Con todo, más allá de la perspectiva que tomemos acerca de las primeras aproximaciones sobre el nacimiento de este tipo de enfoques, la importancia de los estudios no unidisciplinares a inicios del nuevo milenio es evidente, tal como lo plantea el ya citado Thompson (2010):

La interdisciplinariedad se ha convertido en un mantra para el cambio en el siglo XXI. El término aparece en innumerables informes de asociaciones profesionales, organizaciones educativas, organismos de financiamiento, organismos políticos y científicos. Es una palabra clave en los planes estratégicos, acompañada por una retórica de la innovación, la colaboración y la competitividad. (p.1)
Un ejemplo de lo anterior es que la representación de la sociedad por parte de las Ciencias Sociales ya no se corresponde con una máquina o un organismo, sino con conceptos como juego, drama, texto o con el uso de diversos métodos como el análisis de textos y los modelos discursivos para explicar fenómenos sociales (Thompson, 2010, p.25). Ante esto, Nicolescu (1996) explica, que a poco más de un cuarto de siglo de los planteamientos de Piaget, la transdisciplinariedad ha experimentado un florecimiento acelerado en muchas partes del mundo:

[e]n Brasil, Francia, Italia, Canadá, Rumania, Sudáfrica y Suiza; importantes conferencias internacionales dedican sesiones enteras a la transdisciplinariedad en Rusia, Turquía, Canadá, Austria, Estados Unidos, Holanda y otros países; revistas transdisciplinarias son publicadas una tras otra en varias naciones y en la Web, un número sorprendentemente grande de libros sobre transdisciplinariedad fueron publicados en los últimos años, cubriendo una gran diversidad de temas como: educación, estudios sobre ciencia y religión, economía, administración, terapia, geografía, post-colonialismo, nutrición, salud $\mathrm{y}$ ciencias sociales $[\ldots]$ casas editoriales en Francia, Brasil y Rumania fundaron series sobre trasdisciplinariedad, [asimismo] se están dando conferencias en universidades en Estados Unidos, España, Rumania, Francia y Brasil e incluso se están creando cátedras. (p.20) 
Asimismo, Nicolescu (1996) asegura que la transdisciplinariedad se encuentra completamente desarrollada y lista para ser aplicada, toda vez que en el pasado, "[...] nuestras acciones se concentraron en el campo de la educación, un hecho que es natural debido al rol central de la misma en la vida individual y social. Pero ahora tenemos la obligación ética de extender nuestras actividades a los sectores científico, social, político y espiritual" (p. 20).

\section{El diseño desde el enfoque transdisciplinar}

En lo que se refiere a la interacción sujetoobjeto, Llovet (1981) plantea que:
Un problema de diseño no es tanto más complejo cuanto más complicado sea el artefacto, señal u objeto a diseñar [...] un problema de diseño es tanto más complejo cuanto más intrincada sea o pueda ser la red de relaciones contextuales en que se halla o se puede hallar. En este sentido diseñar una locomotora puede ser más laborioso pero no más complejo que diseñar una vivienda unifamiliar; puesto que una locomotora tiene simplemente que ser capaz de jalar un tren, mientras una vivienda tiene que sostener una de las estructuras sociales más críticas y llena de variables que existen en nuestra sociedad

Tabla 1. Principales características de los niveles de interacción disciplinar (Moreno, 2015)

\begin{tabular}{|c|c|c|c|}
\hline $\begin{array}{l}\text { Principales diferencias } \\
\text { entre los niveles de } \\
\text { interacción disciplinar }\end{array}$ & $\begin{array}{c}\text { Orientación } \\
\text { principal }\end{array}$ & Características principales & Forma de evaluación \\
\hline Disciplinariedad & $\begin{array}{l}\text { Generación de } \\
\text { conocimiento }\end{array}$ & $\begin{array}{l}\text {-Objetivo } \\
\text {-Homogéneo } \\
\text {-Paradigmático } \\
\text {-Sujeto/Objeto } \\
\text {-Marcos de investigación comunes }\end{array}$ & $\begin{array}{l}\text {-Revisión de Pares } \\
\text {-Publicación en revistas de la } \\
\text { misma disciplina }\end{array}$ \\
\hline Multidisciplinariedad & $\begin{array}{l}\text { Generación de } \\
\text { conocimiento }\end{array}$ & $\begin{array}{l}\text {-Combinación de diversas disciplinas sin mezclar los } \\
\text { marcos que las componen. } \\
\text { Ejemplo: Los estudios sociales, combinan diversas } \\
\text { disciplinas como lo son la geografía, la ciencia política, } \\
\text { la sociología y la psicología, sin que estas necesariamente } \\
\text { compartan sus marcos disciplinares }\end{array}$ & $\begin{array}{l}\text {-Revisión por especialistas } \\
\text { del área que inició la } \\
\text { investigación } \\
\text {-Publicación en revistas de } \\
\text { la disciplina que inició la } \\
\text { investigación }\end{array}$ \\
\hline Interdisciplinariedad & $\begin{array}{l}\text { Generación de } \\
\text { conocimiento }\end{array}$ & $\begin{array}{l}\text {-Combinación de marcos disciplinares } \\
\text {-Pueden dar lugar a nuevas disciplinas o subdisciplinas } \\
\text { Ejemplo: Bioquímia, Psicología social o ambiental, etc. }\end{array}$ & $\begin{array}{l}\text {-Revisión por especialistas de } \\
\text { diversas disciplinas } \\
\text {-Publicación en revistas de } \\
\text { disciplinas distintas a las del } \\
\text { autor }\end{array}$ \\
\hline Transdisciplinariedad & $\begin{array}{l}\text { Resolución de } \\
\text { problemas }\end{array}$ & $\begin{array}{l}\text {-Aproximación desde diversos saberes, disciplinares y no } \\
\text { disciplinares. Heterogéneo } \\
\text {-No existe un método sino que este se desarrolla según el } \\
\text { desarrollo del problema. Subjetivo } \\
\text {-Necesidad de desarrollar herramientas para la interacción } \\
\text { de los diversos actores. } \\
\text {-Observador implicado }\end{array}$ & $\begin{array}{l}\text {-Verdades contextuales, } \\
\text { basadas en la interpretación } \\
\text {-Difusión en el mismo } \\
\text { momento que se desarrolla el } \\
\text { proyecto. }\end{array}$ \\
\hline
\end{tabular}


actual [la familia] y que marcan el centro de su funcionamiento y avance en muchísimos aspectos: laboral, parental, ocioso, educacional, sexual, estético, psicológico, etcétera. (p.19)

Tradicionalmente, los diseñadores eran educados para trabajar dentro de ciertos marcos disciplinares, de modo que los clientes contrataban a los diseñadores para producir libros, carteles, sillas, refrigeradores, páginas web, interiorismo, edificios, etc. Ahora, cada vez con mayor frecuencia, los diseñadores se encuentran inmersos en proyectos que exigen un cambio en diversos ámbitos: metodológicos, estratégicos, etc., y que en muchas ocasiones se presentan de maneras impredecibles. Todos estos cambios han transformado la manera en que se relacionan los diseñadores con los productores, los consumidores, los usuarios y los ciudadanos, dejando atrás el énfasis en la forma y el proceso [que no por ello han dejado de ser importantes de cierta manera] y se ha inclinado más por la mediación y la facilitación [de instrucciones de operación o juego] que por la creación (Hunt, 2012, p.7).

Actualmente en el desarrollo de los diseños (gráfico, industrial, arquitectónico, de interiores, etc.) es común que sus distintos especialistas trabajen con otros profesionales, es decir, desde perspectivas multidisciplinares. Así, por poner algún ejemplo, es frecuente encontrar a los diseñadores gráficos trabajando en conjunto con impresores, publicistas, redactores, fotógrafos e ilustradores, todos ellos enfocados en un solo problema; de igual modo, los diseñadores industriales trabajan con ingenieros industriales y de producto, con diseñadores gráficos y especialistas en envase y embalaje; los arquitectos con ingenieros civiles, urbanistas y muchos otros actores.

En un primer acercamiento, podríamos decir que las disciplinas proyectuales del diseño se encuentran acostumbradas a la interacción entre diversas disciplinas y actores de la industria de manera al menos multidisciplinaria, pero, cuando nos acercamos un poco más a la manera en que los diseñadores desarrollan sus ideas y productos, se encuentra que los profesionales de estas disciplinas proyectuales trabajan muy a menudo de manera interdisciplinar en distintas etapas de los proyectos. Esto puede observarse, en primera instancia, en la utilización de metodologías flexibles por parte de los diseñadores, ya que son habituados a desarrollar modificaciones metodológicas y a incluir perspectivas teóricas y herramientas propias de otras disciplinas durante las diversas etapas que conforman un proyecto. Por ejemplo, la semiótica y la retórica en el desarrollo de proyectos de diseño gráfico, las teorías sociales y antropológicas en la arquitectura y el diseño industrial y técnicas etnográficas para la recopilación de información en todas ellas (Moreno, 2014, pp.13-14). Esto ha llevado a varios autores a considerar al diseño como una disciplina esencialmente interdisciplinaria Boradkar, 2010; y a nosotros a pensar en la posibilidad de explorar el diseño en la solución de problemas a través de interacciones discipli- 
nares más complejas, como lo es la transdisciplinariedad.

Ahora bien, en el entendido de que las teorías relacionadas con los términos clave hasta aquí descritos: la complejidad, el diseño y la transdisciplinariedad, son relativamente nuevas y que, por ello, aún se encuentran en un proceso de debate y construcción, es necesario resaltar, en este punto, algunas consideraciones acerca de lo que aquí entendemos como diseño transdisciplinar: Primeramente, debemos recordar que la transdisciplinariedad no es el único enfoque existente para la interacción de saberes y que, por ende, no es apropiado para la resolución de cualquier problema; los enfoques disciplinares, multidisciplinares e interdisciplinares son capaces de abordar y resolver una gran diversidad de problemáticas, algunas de ellas de cierta complejidad. En segundo lugar, no todos los proyectos transdisciplinares se pueden abordar a través del diseño, por lo que no se debe pensar que el diseño transdisciplinar puede estudiar cualquier fenómeno. En tal sentido, los proyectos complejos en los que intervengan diversas variables [inseguridad, movilidad, infraestructura, comunicación, etc.] y que se relacionen con la necesidad de encontrar problemas que involucren la conceptualización, proyección y construcción de espacios, ambientes, artefactos o comunicaciones visuales que serán utilizados en conjunto por una diversidad de personas en su cotidianidad, serían los más adecuados para la transdisciplinariedad: ejemplo de ello es el entorno urbano, y más particularmente el espacio público. Asimismo, existe otra cantidad de problemas complejos que incluyen diversas temáticas como la pobreza, la inseguridad, la violencia de género, etc., pero que no tienen que ver con la aplicación directa del diseño. En estas, el diseño puede involucrarse como parte de un equipo transdisciplinar. De igual manera, existen otros problemas complejos de corte transdisciplinar en los que el diseño podría no participar. En tercer lugar, cuando se habla de diseño transdisciplinar, no debe entenderse que el diseño puede resolver el problema por sí mismo, en solitario; pues, como se ha planteado, un problema de esta índole requiere la participación de diversos actores sociales, que participan de acuerdo con el proyecto. Así, cuando se utilice el término transdisciplinar, en realidad se hace referencia a un conjunto de actores sociales con prácticas y saberes diversos, que se encuentran reunidos para resolver un problema complejo. Una cuarta característica del diseño transdisciplinar es la integración de los actores desde la conceptualización del problema $y$, finalmente, las respuestas a las problemáticas abordadas desde este enfoque tendrán que prever la emergencia, la incertidumbre, el caos, en otras palabras, deberán ser evolutivas.

Como anteriormente se ha sugerido, los temas principales aquí presentados, complejidad, transdisciplinariedad y diseño, convergen y se entrelazan, dada su naturaleza, en un espacio común: el entorno humano. Es en este donde intervienen distintos niveles de complejidad, mismos que son "marcos para las actividades" 
(Heskett, 2005, p.102), construidos ingeniosamente a partir de objetos, formas, colores y texturas como elementos constitutivos básicos [usualmente producidos por el diseño], pero que dan lugar, a su vez, a pautas de uso y comportamiento para el trabajo, el ocio y el comercio. Sin duda alguna, así se producen lugares en los que se realizan complejas negociaciones entre la necesidad y el deseo de quienes allí habitan. El diseño, por su naturaleza, se encuentra habituado a trabajar con una orientación hacia el usuario, por lo que el lugar privilegiado en el que se desarrollan las prácticas de diseño es la ciudad o, más específicamente, las grandes urbes, las metrópolis modernas.

En cuanto a la formación de nuevos diseñadores se refiere, Vijay Kumar plantea que los equipos creativos que usualmente utilizan métodos y herramientas tradicionales en el desarrollo y mejora de productos o servicios, no logran un rompimiento o innovación real; por ello explica que para desarrollar lo que él llama una innovación disruptiva, las prácticas y las herramientas existentes simplemente no aplican (2013, p.2). En otras palabras, Kumar plantea la necesidad de crear métodos y herramientas específicas según el proyecto en desarrollo, mismas que deben ser parte integral de la formación de los diseñadores. Para ejemplificar lo anterior, recopila diversos métodos desarrollados por diseñadores para ser aplicados en alguna parte particular del proyecto, por ejemplo: la búsqueda de información en medios populares [como en youtube o facebook] (p.24); el Trends
Matrix, que recopila información sobre cómo las modas afectan la tecnología, los negocios, las personas, la cultura y la política, lo que nos permite observar cómo las tendencias impactan al proyecto (p.38); los mapas de convergencia que evidencian áreas de la vida cotidiana como el trabajo, el hogar, la comunicación móvil, etc., se sobreponen con otras y nuevos comportamientos emergen de ello (p.40); El POEMS, que plantea el estudio de las personas [people], los objetos [objects], el ambiente [environment], los mensajes [messages] y los servicios [services], que nos ayuda a entender un contexto y las relaciones entre los objetos que lo conforman (Kumar, 2013, p.104).

Otros métodos importantes relacionados con las ciencias de la complejidad aplicadas a la generación de información son el systems diagramming, señalado como un método riguroso para entender el contexto y visualizar las relaciones, flujos y disrupciones que se dan entre las diferentes partes de un sistema y entre diferentes sistemas, lo que permite desentrañar lo complejo y entender mejor el problema [teoría de redes]; así como el scalar thinking, el cual implica el desarrollo de un mapa de gradaciones que nos permite observar la naturaleza del problema, los actores y los recursos involucrados al observarlo en diferentes escalas [teoría de fractales] (Hunt, 2012, p.8). Asimismo, otros métodos y técnicas útiles son: la construcción de escenarios, el análisis estadístico, los casos de estudio, la investigación-acción, la lluvia de ideas y el marketing social (Thompson, 2010, p.108). 


\section{Conclusión}

La determinación de las formas [productos del diseño] se ha transformado en una actividad esencial para el desarrollo de la vida en las sociedades contemporáneas. A principios del nuevo milenio, nuestra vida cotidiana y nuestra identidad parecen centrarse en dos pilares: los medios de comunicación y el diseño; ambos tienen una gran presencia en nuestras vidas y se relacionan con todos los ámbitos de nuestra existencia: la economía, la política, la sociedad y la cultura, lo que los convierte en las principales herramientas para la construcción de nuestra identidad como individuos en el siglo XXI. Por tanto, hoy día los productos de diseño no son solo objetos que se adquieren en el mercado, sino un conjunto de pautas culturales que se crean o redes de significación, como las plantea Geertz (2003). Todos somos actores en esa red, ya que en ella personas, cosas e instituciones poseen relevancia y poder de interacción (Latour, 2005).

Desde la década de los cincuenta, el diseño ha demostrado ser no solo una disciplina generadora de objetos, sino que sus alcances y competencias se extienden cada vez más lejos, incluso en áreas que probablemente ni el mismo Gropius en la Bauhaus imaginó, como es el caso del impacto social y económico de los productos, amén de su aplicación como modelo de negocios o su participación en la protección al medio ambiente.

Hoy día, el diseño permite enfrentar escenarios que ayudan a entender las conductas $\mathrm{y}$ necesidades humanas, lo que representa un desafío no solo para las empresas, los gobiernos y las instituciones, sino para los mismos diseñadores en la medida en que deben ayudar a la gente a entender y vivir en un mundo esencialmente complejo. Con otras palabras, es una práctica generadora de lo material y lo inmaterial y su esencia es generativa, especulativa y transformacional.

Por todo lo expresado, el diseño se encuentra en una posición privilegiada para participar en la búsqueda de soluciones para ciertas problemáticas complejas desde perspectivas como la transdisciplinariedad. Esta permite a los diseñadores interesados en participar en este tipo de enfoque la ampliación de sus alcances y oportunidades, tanto en la teoría como en la práctica. Pero también exige, desde el punto de vista formativo, nuevas formas de interacción, que propicien en los diseñadores el desarrollo de habilidades y competencias para abordar proyectos desde visiones no unidisciplinares.

\section{Referencias}

Almarza, F. (2002). La Teoría del Caos. Modelo de Interpretación Epistémica e Instrumento de Solución: Reconciliación entre Ciencias y Humanidades. ESCRITOS, 14 III, 107-150. Recuperado el 27 de julio de 2014 de http://www.pensamientocomplejo.com.ar/docs/files/Fernando $\% 20$ ALmarza-R\%EDsquez $\% 2 \mathrm{C} \% 20 \mathrm{La} \% 20$ Teor $\%$ EDa $\% 20$ de $1 \% 20 \mathrm{Ca}$ os $\% 20$ Modelo\%20de\%20Interpretaci\%F3n.pdf Barabási, A. (2002). Linked. The New Science of Networks. Massachusetts: Perseus Publishing. 
Bertalanffy, L. (1986). Teoría General de los Sistemas: Fundamentos, Desarrollo, Aplicaciones. México: Fondo de Cultura Económica (FCE).

Boradkar, P. (2010). Designing Things: A Critical Introduction to the Culture of Objects. NY: Berg.

Brown, V., Harnis, J., \& Russel J. (2010). Tackling Wicked Problem through the Transdisciplinary Imagination. London: Earthscan.

Deleuze, G. \& Guattari, G. (2002). Mil Mesetas, Capitalismo y Esquizofrenia. España: Pre Textos.

Escobar, A., Hess, D., Licha, I., Sibley, W., Stratehern, M. \& Sutz, J. (2004) “Welcome to Cyberia: Notes on the Anthropology of Cyberculture [and Comments and Reply]." Current Anthropology, 35(3), 211-231.

Espinoza, M. (1995). René Thom: de la Teoría de Catástrofes a la Metafísica. Themata, 14, 321-347.

Feo, R. (2015). Epistemología y práctica de la investigación sobre el aprendizaje estratégico en América Latina. Revista Educación y Humanismo, 17(29), 220-235. http://dx.doi.org/10.17081/ eduhum.17.29.1254.

Flores, A. \& Millán, C. (2002). Desafíos de la Transdisciplinariedad. Bogotá D.C.: Pontificia Universidad Javeriana.

García, L. (2009). Estructuralismo/Posestructuralismo. Recuperado el 05 de mayo de 2015 en http://luisgarciafanlo.blogspot. mx/2009/05/estructuralismo-posestructuralismo-i.html

Geertz, C. (2003). La Interpretación de las Culturas. España: Gedisa.

Heskett, J. (2005). El Diseño en la Vida Cotidiana. Barcelona: Gustavo Gili.

Hunt, J., ed. (2012). Letter from the Editor. Journal of Design Strategies, 5(1) (spring), 5-10.

Kumar, V. (2013). 101 Design Methods. A Structured Approach for Driving Innovation in Your Organization. New Jersey: John Wiley \& Sons.

Kuhn, T. (1962). The Structure of Scientific Revolutions. Chicago: University of Chicago Press.

Latour, B. (2005). Reensamblar lo Social, una Introducción a la Teoría del Actor Red. Buenos Aires: Manantial.

Lyotard, J. (1987). La Condición Postmoderna. Buenos Aires: Cátedra.

Llovet, J. (1981). “Ideología y Metodología del Diseño". Barcelona: Editorial Gustavo Gili.

Maldonado, C. (2009). Complejidad de los Sistemas Sociales: un Reto para las Ciencias Sociales. Cinta Moebio, 36, 146-157.

Maldonado, C. \& Gómez, N. (2010). El Mundo de las Ciencias de la Complejidad. Un Estado del Arte. Bogotá: Universidad del Rosario. Recuperado de www.moebio. uchile.cl/36/maldonado.html

Mandelbrot, B. (1967). How long is the coast of Britain? Statistical self-similarity and 
fractional dimension. Science (156), 636-638

Morin, E. (1994). Introducción al Pensamiento Complejo. Barcelona: Gedisa.

Morin, E. (2004). La Epistemología de la Complejidad. Gazeta de Antropología, 20. Recuperado de http://www.pensamientocomplejo.com.ar/docs/files/MorinEdgar\%20Epistemologia\%20de $\% 20$ la\%20Complejidad.pdf

Moreno, L. (2013). Complejidad, transdisciplinariedad y proyecto: reflexiones sobre los alcances del diseño en el siglo XXI. Taller Servicio 24 Horas, 9(17), 17-25.

Moreno, L. (2014). Complejidad, diseño e indisciplina: nuevas miradas en la práctica y el saber. Taller Servicio 24 Horas, 10 (20 BIS), 5-16.

Moreno, T. (2015). La enseñanza universitaria: una tarea compleja. Revista de Educación Superior, (3), 115-38.

Nicolescu, B. (1996). La Transdisciplinariedad, Manifiesto. Mexíco: Multiversidad Mundo Real. Recuperado el 27 de julio de 2014 de http://www.edgarmorin. org/biografia-edgar-morin/29-tematica/ basarab-nicolescu.html

Nicolescu, B. (2006). Transdisciplinariedad: Pasado, Presente y Futuro (1ra. Parte). Visión Docente con Ciencia, V, 31. Recuperado el 27 de julio de 2014 de http://www.ceuarkos.com/Vision docente/revista31/t3.htm

Outerelo, E. (2000). Teoría de Catástrofes. Números, 43- 44, 253-258. Recuperado de http://www.sinewton.org/numeros/ numeros/43-44/Articulo51.pdf

Payares, L. (2011). Complejidad y Humanidades: Conciliando el Ser y el Devenir. Cuadernos del CENDES, 28(77), 17-42.

Robinson, J. (2008). Being Undisciplined: Transgressions and Intersections in Academia and Beyong. Futures, 40, 70-86. Recuperado de www.researchgate. net/.../3deec52d05a8a41883.pdf

Thompson, J. (1990). Interdisciplinarity: History, Theory and Practice. USA: Wayne University Press.

Thompson, J. (1996). Crossing Boundaries: Knowledge, Disciplinarities, and Interdisciplinarities. USA: Virginia University Press.

Thompson, J. (2001). Transdisciplinarity: Joint Problem Solving among Science, Technology and Society. An Effective Way for Managing Complexity. USA: Springer.

Thompson, J. (2010). Creating Interdisciplinary Campus Cultures: A Model for Strength and Sustainability. USA: Jossey-Bass.

Velázquez, A. (2010). La Organización del Conocimiento en los Modelos Arbóreo, Cartográfico y Reticular. La Indisciplinariedad del Saber: La Multidisciplina en Debate. México: UNAM. 